\title{
Vegetación epifita y captación de neblina en bosques siempreverdes en la Cordillera Pelada, sur de Chile
}

\author{
Epiphytic vegetation and fog precipitation in temperate rainforests \\ of southern Chile's coastal range Cordillera Pelada
}

\author{
Christine Woda $^{1 *}$, Anton Huber ${ }^{2}$, Achim Dohrenbusch ${ }^{1}$ \\ *Autor de correspondencia: ${ }^{1}$ Georg-August-Universität Göttingen, Institut für Waldbau I, Büsgenweg 1, \\ D-37077 Göttingen, Alemania, cwoda@web.de, adohren@gwdg.de \\ ${ }^{2}$ Universidad Austral de Chile, Instituto de Geociencias, Valdivia, Chile, ahuber@uach.cl
}

\begin{abstract}
SUMMARY
This study documents the effect of fog precipitation in a temperate rainforest in Chile's coastal range next to Valdivia and the occurrence of epiphytic groups within different forest types. In 2000, the total amount of rain was $6.966 \mathrm{~mm}$. During the dry summer months, precipitation collected inside the forest exceeded rainfall outside the forest by up to $62 \%$ (93 $\mathrm{mm}$ ) per month due to fog precipitation. In the primary forest (Laurelio-Weinmannietum), the epiphytes have a biomass of $2.2 \mathrm{t} \mathrm{ha} \mathrm{a}^{-1}$ and in a 140 year-old secondary forest (Drimys-Stadium) $2.0 \mathrm{t} \mathrm{ha}^{-1}$. In the 80-year-old secondary forest stand of Fitzroyetum with a very open canopy, the epiphyte biomass reached not more than $0.1 \mathrm{t} \mathrm{ha}^{-1}$. Epiphytic ferns and foliage lichens are more frequently present in darker areas, probably due to more equilibrated conditions of air humidity. In contrast, fruticouse lichens are more frequent in the bright stand of Fitzroyetum, where epiphytic ferns and foliage lichens are nearly absent. These complex interactions between light conditions, air humidity and the ability of stand components to capture fog which influence the abundance of epiphytes should be considered when the forest is managed. Changes in the epiphytic vegetation due to harvesting activities will have strong effects on biodiversity and on a balanced nutrient cycle.
\end{abstract}

Key words: biomass storage, light conditions, Hymenophyllaceae, fruticose lichens, folious lichens.

\section{RESUMEN}

El presente estudio documenta el aporte de agua por intercepción de neblina en bosques siempreverdes de la Cordillera de la Costa cerca de Valdivia y su vegetación epifita. En el año 2000, las precipitaciones alcanzaron 6.966 mm. Durante el verano, las precipitaciones dentro del bosque sobrepasaron a las del campo abierto en un $62 \%(93 \mathrm{~mm})$ debido al adicional aportado por la intercepción de neblina. La biomasa foliar de epifitas y plantas trepadoras alcanzó los $2,2 \mathrm{t} \mathrm{ha}^{-1}$ en el bosque primario de Laurelio-Weinmannietum, y 2,0 t ha ${ }^{-1}$ en el bosque secundario (Drimys-Facies) de 140 años de edad. En un bosque secundario de Fitzroyetum con un dosel relativamente abierto, la biomasa epifítica no sobrepasó las $0,1 \mathrm{t} \mathrm{ha}^{-1}$. Helechos epifitos y líquenes foliosos se ubican preferentemente en áreas umbrófilas. Ello se debe probablemente a la mayor humedad relativa del aire en estos sitios. Al contrario, líquenes fruticosos se encuentran con mayor frecuencia en áreas más soleadas, como en el Fitzroyetum, donde helechos epifitos y líquenes foliosos están ausentes. Las interacciones complejas entre las condiciones de luz, la humedad relativa del aire y la posibilidad del bosque para captar neblina que afectan la abundancia de epifitas deben ser consideradas si se pretende intervenir racionalmente estos bosques. Cambios en la vegetación epifita afectan la biodiversidad de los bosques, balance hidrológico y ciclos biogeoquímicos.

Palabras clave: biomasa, luz, Hymenophyllaceae, líquenes fruticosos, líquenes foliosos.

\section{INTRODUCCIÓN}

Los bosques siempreverdes de la Cordillera de la Costa de Chile forman un ecosistema único en el mundo debido a su especial composición florística. Ellos poseen un alto porcentaje de especies endémicas debido al aislamiento biogeográfico que tiene la zona (Armesto et al. 1997, Villagrán y Hinojosa 1997). El régimen pluviométrico de la zona y el importante aporte de agua por intercepción de neblina, hacen posible que estos bosques se caractericen por una densa vegetación de epifitas, compuesta por musgos, líquenes, helechos y epifitas vasculares. Según Galloway (1995) la biodiversidad de líquenes epifitos de estos bosques se encuentra entre las más altas del mundo.

Actualmente, los bosques siempreverdes de Chile están expuestos a un fuerte deterioro causado por un mane- 
jo no sustentable y una paulatina sustitución por plantaciones forestales (Claro 1998, Wilken 1998, Cisternas et al. 1999). Según WWF (2002) estos bosques pertenecen a las 25 ecorregiones más amenazadas del mundo. En la actualidad no se dispone de mucha información sobre su composición florística y sus requerimientos ecológicos, especialmente el de la vegetación epifítica. Galloway (1995) advierte que el deterioro de estos bosques causa pérdidas invaluables en la biodiversidad de epifitas. También es desconocida la influencia de este grupo de plantas sobre el balance hídrico de los ecosistemas mencionados y el ciclo de nutrientes (Oyarzún et al. 1998, Godoy et al. 2001, Köhler 2002). Por lo tanto, sin tales antecedentes no es posible predecir el efecto de las intervenciones silvícolas en estos ecosistemas en general, ni sobre la vegetación epifita en particular.

El objetivo de este estudio es comparar la abundancia de epifitas (musgos, helechos, líquenes foliosos y fruticosos) y de trepadoras en un bosque primario de Laurelio-Weinmannietum y dos tipos de bosques secundarios (Drimys-Facies del Laurelio-Weinmannietum y Fitzroyetum) en la Cordillera Pelada (Sur de Chile, $40^{\circ} \mathrm{S}$ ). Para caracterizar las condiciones del sitio se analizará, entre otras, la oferta de luz que tiene una fuerte relación con la abundancia de Hymenophyllaceae (Ramírez et al. 1976, Alberdi et al. 1978) y de líquenes. Además, se medirán los aportes de agua por precipitaciones dentro y fuera del bosque y la cantidad proveniente por intercepción de la neblina por un período superior a un año. Este aporte adicional de agua también fue mencionado por otros autores para los bosques templados del sur de Chile (Veblen y Ashton 1982, Pérez et al. 1991, Weathers et al. 2000, Oyarzún et al. 2004).

Este trabajo pretende contribuir a una mejor comprensión de las interacciones ecosistémicas de los bosques siempreverdes de la Cordillera de la Costa y de la importancia que tienen los parámetros abióticos y bióticos en la vegetación epifita. Este conocimiento es fundamental para poder conceptuar planes de manejo que cumplan con las exigencias internacionales sobre la conservación de la biodiversidad (Río 1992 y Johannesburgo 2002).

\section{MÉTODOS}

El estudio se realizó en un bosque primario y en dos bosques secundarios en la Reserva Nacional Valdivia, ubicada en la Cordillera Pelada, X Región, Sur de Chile (40 ${ }^{\circ} \mathrm{S} / 73^{\circ} 8^{\prime} \mathrm{O}, 600-630$ m.s.n.m.). La temperatura promedio anual del área oscila entre $\operatorname{los} 6$ y $9^{\circ} \mathrm{C}$ (15). El suelo es un pseudogley de tipo franco arenoso-limoso derivado de rocas micaesquísticas (Series La Pelada y Hueycolla) con una profundidad media de $50 \mathrm{~cm}$, muy pobre en nutrientes y con una alta saturación de aluminio (Steubing y Godoy 1995, Woda 2004).
El bosque primario pertenece al tipo forestal Laurelio-Weinmannietum según la clasificación de Oberdorfer (1960). El estrato arbóreo superior está formado por Nothofagus nitida (Phil.) Krasser, Weinmannia trichosperma (Cav.) y algunos individuos de Fitzroya cupressoides (Molina). Los estratos inferiores están dominados por Drimys winteri (Forster et Forster), Laurelia philippiana (Looser), Saxegothaea conspicua (Lindl.) y Podocarpus nubigena (Lindl.). Los bosques secundarios pertenecen a Drimys-Facies de Laurelio-Weinmannietum, compuesto por N. nitida y D. winteri, y a Fitzroyetum, donde predominan $D$. winteri y $F$. cupressoides. En el último caso, se trata de un rodal joven que posee una composición florística similar a la de un bosque adulto de Fitzroyetum. Los dos bosques secundarios tienen una estructura de estrato homogéneo. Para estimación de la edad se obtuvieron muestras de leño mediante taladro de incremento, indicando que los individuos son coetáneos (cuadro 1). Por consiguiente, estos bosques se pudieron haber originado después de un gran incendio o una tala rasa. Los bosques primarios en cambio son multietáneos y el análisis de tarugos del árbol de mayor edad analizado $(S$. conspicua), indica que tiene más de 360 años. Un análisis detallado de la composición florística de los bosques investigados se encuentra en Woda (2004).

Características de la vegetación epifita. Para caracterizar la vegetación epifita y de trepadoras se delimitaron en el bosque parcelas de 10 x $10 \mathrm{~m}$. La estructura y la composición arbórea de los bosques secundarios de Drimys-Facies y Fitzroyetum son más homogéneas que las del bosque primario de Laurelio-Weinmannietum. El Fitzroyetum y el Drimys-Facies forman bosques de un solo estrato arbóreo, compuestos por cuatro a seis especies arbóreas, respectivamente, mientras el bosque primario de Laurelio-Weinmannietum representa una estructura multiestratificada con 12 especies arbóreas. Para impedir variaciones que resultan de esta heterogeneidad, se estableció un número de parcelas más grande en el bosque primario (cuadro 1). Las epifitas fueron clasificadas en musgos, líquenes foliosos, líquenes fruticosos y helechos. El último grupo está compuesto por especies del género Hymenophyllum. Primero se determinó la frecuencia relativa con que cada grupo de epifitas estuvo presente en los tres primeros metros de los troncos de cada uno de los árboles. Las trepadoras también fueron consideradas en el análisis. El cuadro 2 muestra las trepadoras encontradas en las parcelas investigadas. Epifitas vasculares, como Bromelia bicolor (Ruiz et Pav.), fueron extremadamente escasas, y por lo tanto no fueron consideradas.

Posteriormente se determinó la biomasa foliar de las epifitas y trepadoras ubicadas en los troncos hasta el inicio de las copas. Para ello se estimó el porcentaje de cobertura que tenían las epifitas en esta sección de cada uno de los troncos. Este valor se calculó para dos zonas en los troncos, la que estaba más expuesta hacia el sol y 
Cuadro 1. Características de los bosques investigados en la Cordillera Pelada, Sur de Chile. La intensidad relativa de luz fue medida a $0,5 \mathrm{~m}$ sobre el suelo. (DAP = Diámetro altura de pecho). *Edad máxima de los árboles analizados (en paréntesis coeficiente de variación).

Stand characteristics of studied forest plots in the Cordillera Pelada (South Chile). Light intensity was measured $0.5 \mathrm{~m}$ above ground level. (DAP = Diameter in breath hight). *Maximal age of tree samples.

\begin{tabular}{|c|c|c|c|c|c|c|c|c|}
\hline Tipo de bosque & $\begin{array}{l}\text { Número de } \\
\text { parcelas } \\
\left(100 \mathrm{~m}^{2}\right)\end{array}$ & $\begin{array}{l}\text { Edad del } \\
\text { rodal } \\
\text { (años) }\end{array}$ & $\begin{array}{l}\text { Área basal } \\
\left(\mathrm{m}^{2} \mathrm{ha}^{-1}\right)\end{array}$ & $\begin{array}{l}\text { Volumen } \\
\text { fustal } \\
\left(\mathrm{m}^{3} \mathrm{ha}^{-1}\right)\end{array}$ & $\begin{array}{l}\text { Número de } \\
\text { árboles ha }{ }^{-1} \\
>5 \mathrm{~cm} \mathrm{DAP}\end{array}$ & $\begin{array}{c}\text { Altura del } \\
\text { estrato arbóreo } \\
\text { superior (m) }\end{array}$ & $\begin{array}{c}\text { Intensidad } \\
\text { relativa de } \\
\text { luz } \\
(\%)\end{array}$ & $\begin{array}{c}\text { Biomasa } \\
\text { foliar de } \\
\text { epifitas y } \\
\text { trepadoras } \\
\left(\mathrm{t} \mathrm{ha}^{-1}\right)\end{array}$ \\
\hline $\begin{array}{l}\text { Laurelio- } \\
\text { Weinmannietum } \\
\text { (bosque primario) }\end{array}$ & 18 & $360 *$ & $\begin{array}{r}108 \\
( \pm 38 \%)\end{array}$ & $\begin{array}{r}831 \\
( \pm 39 \%)\end{array}$ & $\begin{array}{r}1.771 \\
( \pm 45 \%)\end{array}$ & 25 & 7,3 & 2,0 \\
\hline $\begin{array}{l}\text { Drimys-Facies } \\
\text { del Laurelio- } \\
\text { Weinmannietum } \\
\text { (bosque secundario) }\end{array}$ & 7 & aprox. 140 & $\begin{array}{r}88 \\
( \pm 21 \%)\end{array}$ & $\begin{array}{r}761 \\
( \pm 20 \%)\end{array}$ & $\begin{array}{r}1.020 \\
( \pm 25 \%)\end{array}$ & 30 & 6,8 & 2,2 \\
\hline $\begin{array}{l}\text { Fitzroyetum } \\
\text { (bosque secundario) }\end{array}$ & 5 & aprox. 80 & $\begin{array}{r}38 \\
( \pm 19 \%)\end{array}$ & $\begin{array}{r}196 \\
( \pm 19 \%)\end{array}$ & $\begin{array}{r}2.677 \\
( \pm 28 \%)\end{array}$ & 10 & 20,1 & 0,1 \\
\hline
\end{tabular}

Cuadro 2. Especies trepadoras en los bosques de LaurelioWeinmannietum, Drimys-Facies y Fitzroyetum en la Cordillera Pelada, Sur de Chile. El ordenamiento está hecho según su frecuencia (de mayor a menor).

Vine species in studied forest plots of Laurelio-Weinmannietum, Drimys-Facies and Fitzroyetum in Cordillera Pelada, South Central Chile. Species are arranged from higher to lower frequency.

\begin{tabular}{|c|c|}
\hline Tipo de bosque & Trepadoras \\
\hline $\begin{array}{l}\text { Laurelio-Weinmannietum } \\
\text { (bosque primario) }\end{array}$ & $\begin{array}{l}\text { Philesia magellanica (JF } \\
\text { Gmel), Asteranthera ovata } \\
\text { (Cav.) Hanst., Campsidium } \\
\text { valdivianum (Phil.) Skottsb., } \\
\text { Pseudopanax laetevirens } \\
\text { (Gay) Harms., Mitraria } \\
\text { coccinea Cav., Griselinia } \\
\text { ruscifolia (Clos) Taub., } \\
\text { Elyotropus chilensis (A.DC.) } \\
\text { Muell.-Arg., Luzuriaga } \\
\text { polyphylla (Hook.) Macbr., } \\
\text { Griselinia racemosa (Phil.) } \\
\text { Taub. }\end{array}$ \\
\hline $\begin{array}{l}\text { Drimys-Facies } \\
\text { del Laurelio-Weinmannietum } \\
\text { (bosque secundario) }\end{array}$ & $\begin{array}{l}\text { Asteranthera ovata, } \\
\text { Luzuriaga polyphylla, } \\
\text { Mitraria coccinea, Griselinia } \\
\text { racemosa (Phil.) Taub., } \\
\text { Griselinia ruscifolia, } \\
\text { Campsidium valdivianum, } \\
\text { Philesia magellanica, } \\
\text { Elytropus chilensis, } \\
\text { Pseudopanax laetevirens. }\end{array}$ \\
\hline $\begin{array}{l}\text { Fitzroyetum } \\
\text { (bosque secundario) }\end{array}$ & $\begin{array}{l}\text { Philesia magellanica, } \\
\text { Campsidium valdivianum, } \\
\text { Pseudopanax laetevirens, } \\
\text { Luzuriaga polyphylla, } \\
\text { Griselinia racemosa. }\end{array}$ \\
\hline
\end{tabular}

la del lado opuesto a ésta, respectivamente. Al multiplicarse el valor promedio de estas coberturas por la superficie total del tronco se obtuvo la superficie del tronco cubierta por las epifitas.

A continuación se determinó el valor promedio de la biomasa foliar de las epifitas y trepadoras correspondientes a una superficie de muestreo de $10 \times 10 \mathrm{~cm}$. Para este cálculo se utilizaron 20 muestras por bosque. Al multiplicar este valor por la superficie de los troncos cubiertos con epifitas, se estableció la cantidad total de fitomasa (peso seco x unidad de superficie) de éstas para cada uno de los bosques. Debido a que no se consideraron las epifitas y plantas trepadoras ubicadas en las copas de los árboles, el valor total de la fitomasa es subestimado, sobre todo considerando que las plantas trepadoras generalmente desarrollan su mayor biomasa foliar en el área de las copas. Aunque no se observó mayor presencia de epifitas en las copas de los árboles, la excepción se registró en $P$. nubigena. Además, se debe considerar que la biomasa de los tallos de las plantas trepadoras no fue levantada.

Radiación fotosintética activa. Para medir la radiación fotosintética activa (PAR) se instalaron radiómetros construidos en el Instituto Forestal de la Universidad Göttingen, Alemania (Dohrenbusch et al. 1995). Los instrumentos fueron instalados en el centro de cada parcela (representando un área de $100 \mathrm{~m}^{2}$ ) a una altura de $0,5 \mathrm{~m}$ sobre el suelo. Los registros simultáneos en sitios abiertos fuera del bosque permitieron determinar la fracción de la radiación total que llegó al interior de los bosques (intensidad relativa).

Precipitaciones. Las precipitaciones fueron medidas dentro y fuera del bosque Laurelio-Weinmannietum, con 
pluviómetros construidos con tubos de PVC (1 m de largo, $10 \mathrm{~cm}$ de diámetro) (Grunow 1955, Steubing et al. 2002). Según Grunow (1955), el balance hídrico para sitios con neblina es [1]:

$$
\mathrm{L}+\mathrm{N}=(\mathrm{D}+\mathrm{E})-\left(\mathrm{I}_{\mathrm{L}}+\mathrm{I}_{\mathrm{N}}\right)
$$

donde $\mathrm{L}=$ precipitaciones de lluvia, $\mathrm{N}=$ precipitaciones adicionales por intercepción de neblina, $\mathrm{D}=$ precipitación directa, $\mathrm{E}=$ escurrimiento fustal, $\mathrm{I}_{\mathrm{L}}$ e $\mathrm{I}_{\mathrm{N}}=$ pérdidas de agua por evaporación de las precipitaciones retenidas en el dosel y de la neblina interceptada por la biomasa aérea del bosque, respectivamente.

En los bosques investigados una densa vegetación epifita en los troncos intercepta el escurrimiento fustal y lo transforma en precipitación directa para alcanzar el suelo (Köhler 2002, Huber y Martínez 1995, Huber e Iroumé 2001). Según la teoría de Grunow, la precipitación total que llega al suelo de los bosques puede exceder a la registrada fuera de él porque el agua aportada por la neblina puede sobrepasar las pérdidas por intercepción del dosel.

Para probar esta hipótesis se instaló, junto a los pluviómetros, un captador de neblina según Grunow (1955) que consiste en una malla metálica que se instala en forma cilíndrica encima de los tubos. Las gotas filtradas por la malla corren hacia el tubo, lo que permite determinar la fracción de agua que alcanza el suelo por intercepción de la neblina en conjunto con las precipitaciones.

\section{RESULTADOS}

Biomasa y abundancia de epifitas. En el bosque primario de Laurelio-Weinmannietum y en el bosque secundario de Drimys-Facies las epifitas y plantas trepadoras tienen una biomasa foliar de $2 \mathrm{t} \mathrm{ha}^{-1}$ (cuadro 1). En los tres metros inferiores de los troncos de los árboles, las epifitas más abundantes son los musgos, líquenes foliosos y helechos (figura 1). La presencia de trepadoras también es frecuente, pero poseen una menor participación en el peso de la biomasa que las epifitas debido al reducido tamaño de sus hojas a lo largo de los troncos. No obstante, se debe considerar que las trepadoras alcanzan generalmente una alta biomasa con sus tallos y su masa foliar en las copas de los árboles, que no fue considerada en este estudio.

Una situación totalmente diferente se produce en el bosque secundario de Fitzroyetum, donde los líquenes fruticosos tienen una alta abundancia, y la biomasa foliar de las epifitas no sobrepasa las $0,1 \mathrm{t} \mathrm{ha}^{-1}$, siendo mucho menor que en los otros bosques.

La abundancia de las epifitas en el bosque primario de Laurelio-Weinmannietum y en el bosque secundario de Drimys-Facies varía según las especies arbóreas, probablemente debido a las características de la corteza y a

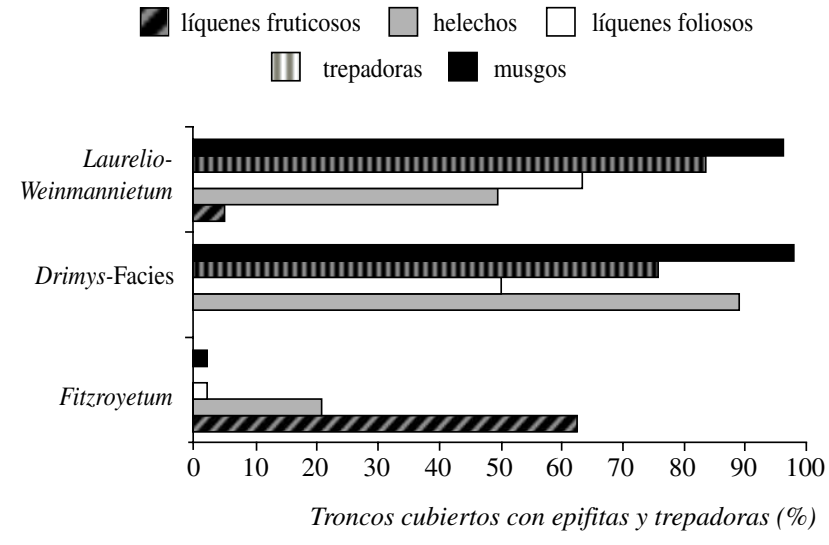

Figura 1. Abundancia de las epifitas y trepadoras en los tres metros inferiores de los troncos de los árboles $>7 \mathrm{~cm}$ DAP en un bosque primario de Laurelio-Weinmannietum, un bosque secundario de Drimys-Facies y un bosque secundario de Fitzroyetum en la Cordillera Pelada del Sur de Chile

Frequency of epiphytes and vines on the first 3 meters of tree stems $(>7 \mathrm{~cm} \mathrm{DBH})$ a primary forest stand of LaurelioWeinmannietum and secondary forest stands of Drimys-phase and Fitzroyetum in the coastal range Cordillera Pelada (South Chile).

la permeabilidad del dosel para dejar pasar la luz (cuadro 3). De todos los árboles, P. nubigena y L. philippiana muestran la cobertura más densa de epifitas, extendiéndose hasta la copa de los árboles. Ambas especies poseen un dosel denso que da mucha sombra. Además, tienen una corteza muy rugosa y porosa, que presumiblemente puede retener bastante agua. Las cortezas de W. trichosperma y $N$. nitida, que están menos cubiertas de epifitas, tienen características similares. Debido a que el dosel de estas especies es más permeable a la luz, los troncos reciben una mayor cantidad de radiación solar directa. Esta situación probablemente limita el crecimiento de los helechos poiquilohidros del género Hymenophyllum, que tienen poca presencia en este tipo de árboles.

Saxegothaea conspicua es un árbol que tiene una copa densa y posee un tronco cubierto con una corteza dura y lisa que se desprende en placas. Estas condiciones posiblemente limitan el crecimiento de los helechos en esta especie. Finalmente, D. winteri, la especie arbórea más abundante en los bosques investigados, muestra la menor presencia de epifitas. Solamente los musgos están frecuentemente presentes. Drimys winteri tiene una corteza dura, con un $\mathrm{pH}$ relativamente bajo y que además contiene químicos (taninos, alcaloides y otras sustancias) que protegen a la madera contra hongos e insectos (Hall y Witte 1998), los que también podrían inhibir el crecimiento de los helechos y líquenes foliosos.

Las plantas trepadoras son frecuentes en todas las especies arbóreas. Como estas plantas son vasculares, se nutren directamente del suelo, es decir, son menos dependientes de la humedad relativa del aire y de la humedad del tronco que las epifitas poiquilohidros. En consecuencia, su mayor abundancia se observa en la superficie 
de los troncos que reciben más radiación solar directa (noreste). Al contrario, las epifitas, especialmente los helechos y líquenes foliosos, son más abundantes en el lado más protegido de los troncos de la radiación solar (sureste), donde supuestamente la humedad de la superficie es mayor.

Condiciones de luz. La localización de los líquenes y los helechos en los troncos muestra una fuerte asociación con la oferta de luz. Helechos y líquenes foliosos se encuentran preferentemente en sitios más umbrófilos, mientras que los líquenes fruticosos lo hacen en las zonas más iluminadas (figura 2). En el rodal de Fitzroyetum, que recibe más radiación solar (cuadro 1 ), los líquenes fruticosos son los más abundantes, mientras que los helechos y líquenes foliosos son poco frecuentes. Ellos predominan principalmente en el bosque primario de LaurelioWeinmannietum y en el bosque secundario de DrimysFacies (figura 1). Allí, con frecuencia, el lado sombreado de los troncos está más cubierto por epifitas que el lado más expuesto a la radiación solar. La abundancia de los musgos y las trepadoras no muestra una dependencia con la intensidad de la luz. No obstante, en el rodal del Fitzroyetum, que registra la mayor luminosidad, prácticamente no están presentes (figura 2).

En general, la cobertura de epifitas y trepadoras en los troncos sobrepasa el $50 \%$ en sitios con una luminosidad relativa cercana al $5 \%$ (figura 3 ). En las partes más claras, con una luminosidad relativa de hasta $35 \%$, el grado de cobertura es inferior al 5\%. Se destaca nuevamente el rodal de Fitzroyetum con la menor cobertura de epifitas. Entre los rodales del bosque secundario de Drimys-Facies y del bosque primario de Laurelio-Weinmannietum no se observan diferencias.
Condiciones pluviométricas. Una densa vegetación de epifitas es típica de bosques de neblina ubicados en zonas de climas húmedos (24). En el año 2000 las precipitaciones en el área de estudio fueron extremamente altas, alcanzando los $6.966 \mathrm{~mm}$. Además, la distribución de las precipitaciones durante el año fue muy heterogénea. En los meses invernales de junio y julio se produjo más de la mitad de la precipitación anual, mientras que en los meses de verano se registró sólo un $9 \%$. Con excepción del mes de junio, las precipitaciones medidas dentro del bosque primario de Laurelio-Weinmannietum sobrepasaron a las del exterior del bosque (cuadro 4).

La mayor precipitación dentro del bosque se debió al aporte adicional de agua por intercepción de la neblina. Durante el periodo de marzo 2000 a marzo 2001, de un total de 112 registros realizados en un pluviómetro con neblinómetro, un $89 \%$ de ellos tuvieron un valor superior a los de los pluviómetros sin neblinómetro. Sólo un $8 \%$ de los aportes de agua por neblina sucedieron en períodos sin precipitaciones. Los mayores aportes relativos de agua por neblina se registraron en días con poca lluvia (figura 4). Según lo observado por los autores, la neblina en la Cordillera Pelada se forma cerca de la superficie, cuando masas húmedas de aire provenientes del Pacífico son dirigidas contra las laderas de la Cordillera y obligadas a ascender (neblina orográfica).

El neblinómetro dentro del bosque no registró un aporte superior de agua que los pluviómetros dentro del bosque. Ello se explica porque la neblina previamente interceptada por el dosel alcanzó el suelo como precipitación.

Los aportes relativos de agua por intercepción de neblina registraron sus máximos durante los meses con menores precipitaciones en verano, sobrepasando en has-

Cuadro 3. Abundancia de trepadoras y epifitas en especies arbóreas en un bosque primario de Laurelio-Weinmannietum y un bosque secundario de Drimys-Facies de Laurelio-Weinmannietum en la Cordillera Pelada en el Sur de Chile. Los valores indican el porcentaje de árboles cubiertos con epifitas en los 3 metros inferiores de los troncos. Valores de pH según Albin (1975).

Frequency of vines and epiphytes on trees in a primary forest stand of Laurelio-Weinmannietum and a secondary forest stand of Fitzroyetum in Cordillera Pelada, South Chile. The values show the percentage of trees $(>7 \mathrm{~cm} \mathrm{DBH})$ covered by epiphytic plants on the first 3 meters of stem. pH-values following Albin (1975).

\begin{tabular}{|c|c|c|c|c|c|c|c|}
\hline \multirow[b]{2}{*}{ Especie arbórea } & \multirow[b]{2}{*}{ Estructura de la corteza } & \multirow[t]{2}{*}{$\begin{array}{l}\mathrm{N}^{\mathrm{o}} \mathrm{de} \\
\text { troncos }\end{array}$} & \multicolumn{5}{|c|}{$\begin{array}{l}\text { Troncos cubiertos con } \\
\text { epifitas }(\%)^{*}\end{array}$} \\
\hline & & & $\mathrm{m}$ & $\mathrm{h}$ & lfo & If & $\mathrm{t}$ \\
\hline Podocarpus nubigena & Se desprenden en lonjas, $\mathrm{pH}: 5$ & 22 & 91 & 91 & 86 & - & 77 \\
\hline Laurelia philippiana & Porosa, blanda, $\mathrm{pH}: 5,1$ & 17 & 100 & 88 & 71 & - & 94 \\
\hline Weinmannia trichosperma & Porosa, blanda, $\mathrm{pH}: 4,6$ & 16 & 100 & 63 & 63 & - & 94 \\
\hline Nothofagus nitida & Similar encino, dura & 34 & 94 & 68 & 85 & - & 76 \\
\hline Saxegothaea conspicua & Lisa, se desprende en placas & 38 & 97 & 47 & 84 & 3 & 89 \\
\hline Drimys winteri & Lisa y dura, $\mathrm{pH}: 3,8$ & 71 & 99 & 46 & 27 & 10 & 77 \\
\hline
\end{tabular}

* $\mathrm{N}^{\mathrm{o}}$ : número de troncos, m: musgos, h: helechos, lfo: líquenes foliosos, lf: líquenes fruticosos, t: trepadoras. 

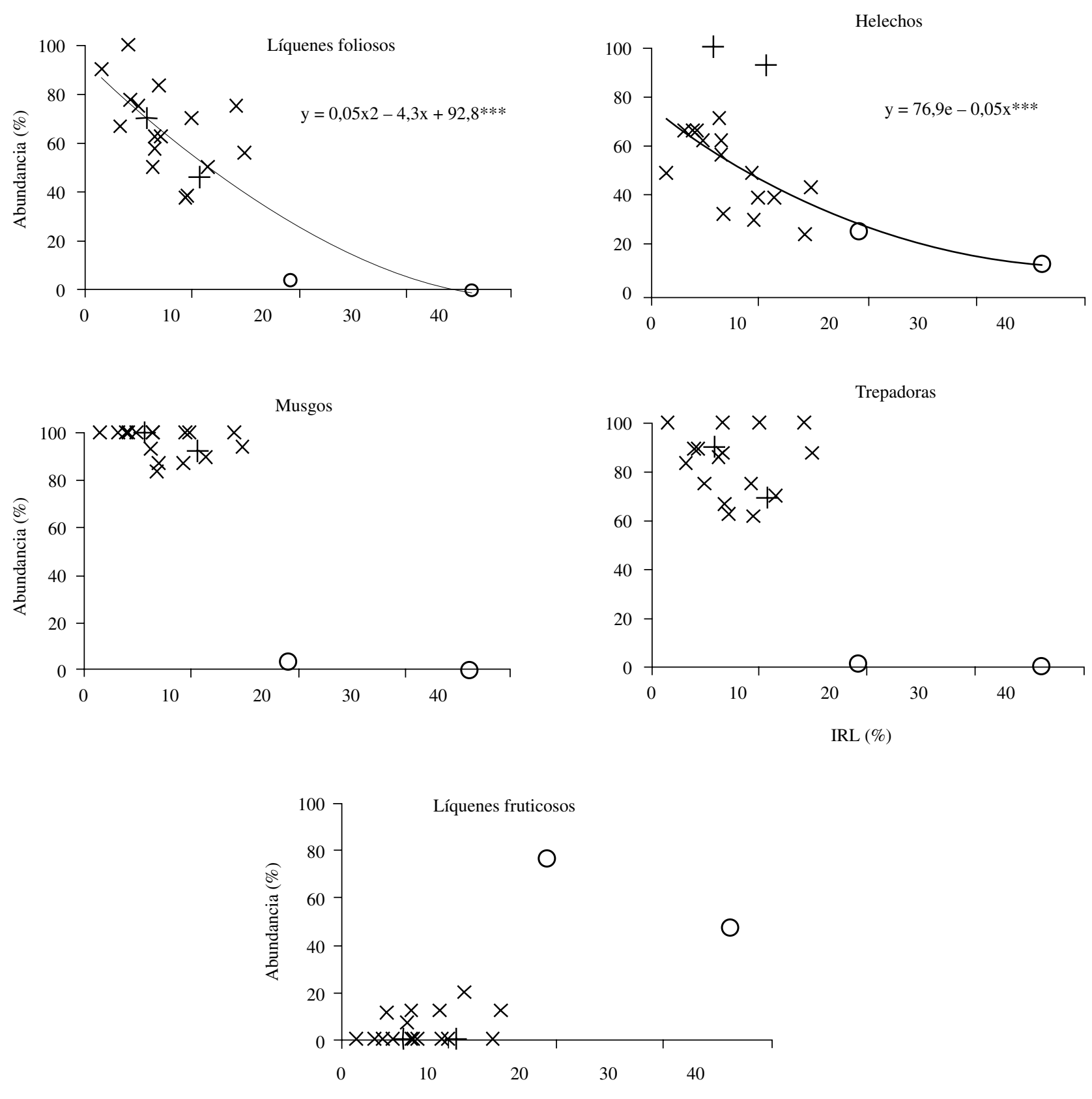

$\operatorname{IRL}(\%)$

Figura 2. Abundancia relativa (\%) de árboles cubiertos por helechos (Hymenophyllaceae), líquenes fruticosos, líquenes foliosos, musgos y trepadoras en los 3 metros inferiores de sus troncos en parcelas de $100 \mathrm{~m}^{2}$ en relación a la intensidad relativa de luz (IRL). Las parcelas están ubicadas en un bosque primario de Laurelio-Weinmannietum (x) y en bosques secundarios de DrimysFacies (+) y de Fitzroyetum (o). La IRL fue medida en el centro de cada parcela. (***: $P<0,001$ ).

Percentage of frequency of trees covered by epiphytic ferns (Hymenophyllaceae), folious and fruticous lichens, mosses and vines on the first 3 meters of stems (> $7 \mathrm{~cm} \mathrm{DBH}$ ) in $100 \mathrm{~m}^{2}$ plots in relation to relative light intensity (IRL). The sample plots are located in a primary forest stand of Laurelio-Weinmannietum (x) and in secondary forest stands of Drimys-Phase (+) and Fitzroyetum (o). The light intensity was measured in the center of each sample plot.

ta un $62 \%$ a las precipitaciones a campo abierto en diciembre (figura 5). Cuando las lluvias superaron los 500 $\mathrm{mm} \mathrm{mes}^{-1}$, como las reportadas para el período mayo- septiembre, esta relación disminuyó. Para el mes de junio, las precipitaciones dentro del bosque fueron $26 \%$ inferiores a las de afuera. 


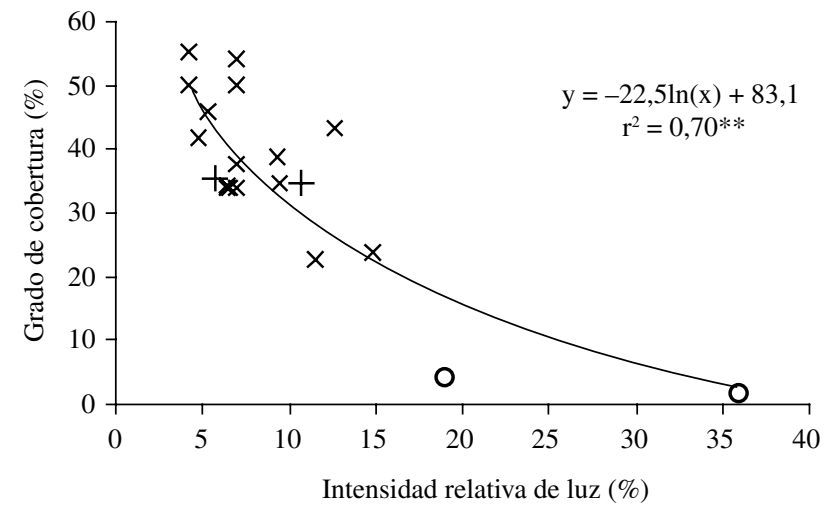

Figura 3. Promedio del grado de cobertura de epifitas y trepadoras $(\%)$ en troncos de árboles $>7 \mathrm{~cm}$ DAP en parcelas de $100 \mathrm{~m}^{2}$ en un bosque primario de Laurelio-Weinmannietum (x) y bosques secundarios de Drimys-Facies (+) y de Fitzroyetum (o) en relación a la intensidad relativa de luz. La IRL fue medida en el centro de cada parcela. (**: $P<0,01)$.

Average of percentage of coverage of epiphytic species and vines on tree stems $>7 \mathrm{~cm} \mathrm{DBH}$ in $100 \mathrm{~m}^{2}$ plots in relation to relative light intensity (IRL) in a primary forest stand of LaurelioWeinmannietum (x) and secondary forest stands of Drimys-Phase (+) and Fitzroyetum (o). Light intensity was measured in the center of each sample plot.

\section{DISCUSIÓN}

Una densa vegetación epifita es típica para bosques montanos ubicados en zonas lluviosas con alta humedad relativa durante todo el año (Stadtmüller 1987). Las precipitaciones anuales en la Cordillera Pelada se suponían entre los 3.000 y $5.000 \mathrm{~mm}$ (Alberdi 1966, Hueck 1966, Huber 1975, Oyarzún et al. 1998, Huber e Iroumé 2001). En el presente trabajo se registraron precipitaciones cercanas a los $7.000 \mathrm{~mm}$ año ${ }^{-1}$. El año del estudio (2000) fue excepcionalmente lluvioso en todo Chile, por consiguiente, esta cantidad debe considerarse como un valor extremo.

El balance hídrico del área del estudio está fuertemente influido por los aportes de agua por neblina. Entre febrero 2000 y enero 2001, las precipitaciones dentro del bosque de Laurelio-Weinmannietum fueron sólo $1 \%$ inferior a las de fuera del bosque. Ello indica que casi todas las pérdidas de agua por intercepción del dosel

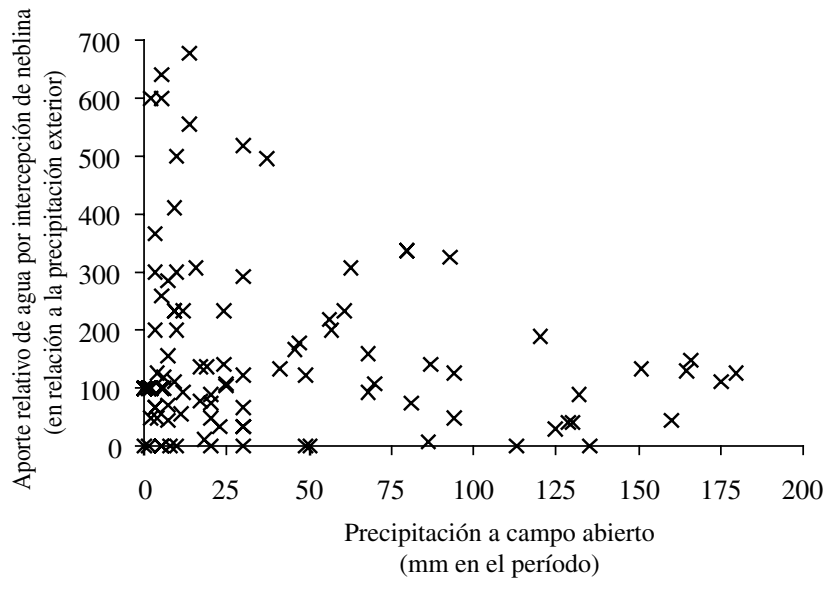

Figura 4. Cantidad de agua captada en un neblinómetro (según Grunow 1955) desde marzo de 2000 a marzo de 2001 en la Cordillera Pelada, Sur de Chile. Los valores indican su equivalencia con respecto a la precipitación total del correspondiente período.

Amount of water collected by a fog catcher (model of Grunow, 1955) between March 2000 and March 2001 in the coastal range Cordillera Pelada, South Chile. Values of fog precipitation are given in relation to rainfall on open field during the same period.

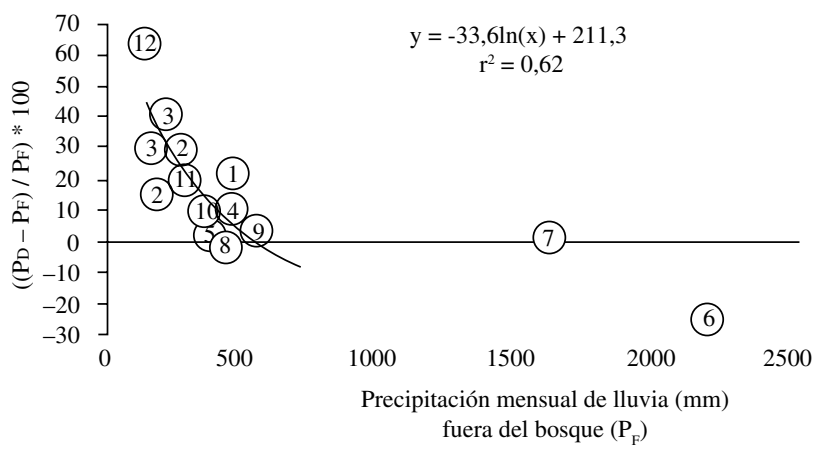

Figura 5. Relación entre la precipitación mensual registrada dentro del bosque de Laurelio-Weinmannietum $\left(\mathrm{P}_{\mathrm{D}}\right)$ y fuera del bosque $\left(\mathrm{P}_{\mathrm{F}}\right)$. Los números indican el mes (1-12 corresponden a enero a diciembre). Valores positivos: Aporte adicional de agua por la intercepción de neblina.

Relationship between monthly precipitation within a primary forest stand of Laurelio-Weinmannietum $\left(\mathrm{P}_{\mathrm{D}}\right)$ and amount of precipitation on open field condition $\left(\mathrm{P}_{\mathrm{F}}\right)$. Numbers indicate the respective month: 1 = January, 2 = February. Positive values: fog precipitation as a result of interception processes.

Cuadro 4. Precipitaciones mensuales ( $\mathrm{mm}$ ) dentro y fuera de un bosque primario de Laurelio-Weinmannietum en la Cordillera Pelada en el Sur de Chile (enero 2000 a marzo 2001).

Monthly precipitation (mm) from February 2000 to March 2001 within and outside a primary forest stand of Laurelio-Weinmannietum in the coastal range of Cordillera Pelada (South Chile). s.i. = without information.

\begin{tabular}{llllllllllllllll}
\hline & \multicolumn{1}{c}{} & \multicolumn{1}{c}{ año 2000} & \multicolumn{1}{c}{ año 2001} \\
Sitio & E & F & M & A & M & J & J & A & S & O & N & D & E & F & M \\
\hline Fuera & 170 & 272 & 172 & 451 & 381 & 2.160 & 1.600 & 432 & 537 & 361 & 287 & 143 & 453 & 187 & 223 \\
Bosque & s.i. & 350 & 223 & 494 & 385 & 1.599 & 1.609 & 422 & 552 & 395 & 342 & 233 & 546 & 214 & 312 \\
\hline
\end{tabular}

s.i.: sin información. 
fueron compensadas por el aporte adicional por intercepción de la neblina. En otros bosques siempreverdes en la Cordillera de la Costa sin influencia de neblina, las pérdidas por intercepción alcanzaron el $20 \%$ de la precipitación anual (Echeverría 1994, Fuente et al. 1994, Huber y Martínez 1995, Oyarzún et al. 1998, Huber e Iroumé 2001). Si se considera que las precipitaciones del período febrero 2000 a enero 2001 fueron de $7.249 \mathrm{~mm}$, se podría inferir que las pérdidas de agua por intercepción podrían ser cercanas a $1.000 \mathrm{~mm}$; por consiguiente, esta cantidad debió ser contrarrestada por la neblina interceptada.

La importancia de la neblina para la vegetación en la Cordillera de la Costa está bien investigada para el norte de Chile (Aravena et al. 1989, Cereceda y Schemenauer 1991). Para la zona lluviosa del sur no hay mayores investigaciones al respecto. Inicialmente, Veblen y Ashton (1982), y Pérez et al. (1991) mencionan aportes adicionales de agua por neblina en los bosques templados en la Cordillera de la Costa en el sur de Chile sin cuantificarlos. Oyarzún et al. 2004 reportan de precipitaciones de neblina en un bosque siempreverde en la Cordillera de los Andes en el sur de Chile, y Weathers et al. (2000) han registrado precipitaciones de neblina en la isla de Chiloé.

A pesar de la enorme cantidad de lluvia anual en la Cordillera Pelada, pueden ocurrir períodos de sequía en el verano. Ello se debe a la heterogénea distribución anual que tienen las precipitaciones. En el año 2000 sólo un $9 \%(630 \mathrm{~mm})$ de la precipitación total se registró durante los meses de verano. Períodos de sequía estival también son mencionados para otros bosques en el sur de Chile con precipitaciones anuales de $3.000 \mathrm{~mm}$ (Echeverría 1994) y $2.200 \mathrm{~mm}$ (Figueroa y Castro 2000). Debido a las características del régimen pluviométrico, muchas plantas vasculares de los bosques de esta zona poseen propiedades xerofíticas para reducir la transpiración (Weinberger et al. 1973, Weinberger 1974). Las epifitas en cambio son poiquilohidras y por lo tanto no se pueden proteger de la deshidratación. Especialmente los helechos epifitos del género Hymenophyllaceae son muy sensibles a los cambios de humedad relativa de aire, por no tener una vacuola central.

En esto la neblina juega un rol importante como una fuente adicional de agua para la vegetación, especialmente para las epifitas poiquilohidras. En el área de estudio, los aportes relativos de agua de la neblina fueron máximos en los meses más secos de verano, alcanzando hasta un $62 \%$ en diciembre con respecto a las precipitaciones a campo abierto. Durante el invierno, cuando las precipitaciones superaron los $500 \mathrm{~mm}$ por mes como las que fueron reportadas para el período mayo-septiembre, su participación relativa en el aporte total de agua disminuyó. Considerando la poca precipitación por lluvia en los meses del verano y la sensibilidad de las epifitas en cuanto a la humedad relativa del aire y la humedad de la superficie de los troncos donde crecen, se puede clasificar la precipitación de neblina en el verano como un aporte significativo para sostener la diversidad de las epifitas durante este período crítico. La importancia de precipitaciones de neblina para la vegetación en épocas de sequía en zonas climáticas con alta humedad también fue postulada para bosques húmedos tropicales de Venezuela (Vogelmann 1973), para bosques de neblina en México (Stadtmüller 1987) y bosques siempreverdes de la costa de América del Norte (Dawson 1998).

La sensibilidad de las epifitas con respecto al balance hídrico se manifiesta también en su asociación con las condiciones de la luz. Entre las epifitas, los helechos (Hymenophyllaceae) y líquenes foliosos son más frecuentes en sitios oscuros. Una situación similar es reportada para las Hymenophyllaceae de otros bosques siempreverdes de Chile (Ramírez et al. 1976, Armesto et al. 1997). En el área del estudio, ambas formas de vida tienen su mayor abundancia en el bosque primario de LaurelioWeinmannietum y en el bosque secundario de DrimysFacies (edad de 140 años), donde las condiciones de luz son semejantes, alcanzando 7\% de IRL. En estos tipos de bosques la vegetación epifita forma una biomasa foliar de $2 \mathrm{t} \mathrm{ha}^{-1}$ sin la consideración de la biomasa en las copas de los árboles. Una biomasa mayor de epifitas (4 de $5 \mathrm{t} \mathrm{ha}^{-1}$ ) ha sido estimada para el rodal de un bosque primario del tipo siempreverde en Chiloé (Pérez et al. 1991). En el bosque secundario de Fitzroyetum (edad de 80 años), que recibe más luz (20\% IRL), la biomasa de las epifitas solamente alcanza $0,1 \mathrm{t} \mathrm{ha}^{-1}$. Allí crecen sólo líquenes fruticosos, que tienen una gran demanda de luz, lo que indica que son más resistentes a la radiación solar (Galloway 1995, Wirth 1995). En general, la humedad relativa del aire al interior de bosques es menor que en los lugares más soleados (Huber et al. 1986). Además, debido a la estructura del bosque de Fitzroyetum, que representa un solo estrato arbóreo de 10 metros de altura máxima, se puede asumir un aporte menor de precipitaciones de neblina que en el bosque multiestrato de Laurelio-Weinmannietum. La ausencia de los helechos y los líquenes foliosos en el bosque secundario de Fitzroyetum son indicadores que revelan la sensibilidad a los cambios de humedad relativa del aire que ellos poseen.

Las epifitas juegan un rol importante en el ciclo de nutrientes en los bosques (Bruijnzeel y Veneklass 1998). Ello se debe por un lado al aumento de la superficie para la intercepción de la neblina. Oyarzún et al. (2004) reportan precipitaciones de neblina con aportes en ciclos biogeoquímicos significativos en un bosque siempreverde en la Cordillera de los Andes, y en Weathers et al. (2000) han sido registrados flujos de nitrógeno vía neblina en la isla de Chiloé. Por otro lado, varios autores sugieren la importancia de la fijación de nitrógeno por líquenes y epifitas en bosques del sur de Chile (Guzmán et al. 1990, Oyarzún et al. 1998, Godoy et al. 2001). Estas consecuencias en el balance de nutrientes son de 
mayor importancia para los bosques que están establecidos en suelos con bajo contenido de nutrientes, que es una característica de los suelos de esta zona de la Cordillera de la Costa (Woda 2004).

Los resultados revelan lo complejas que son las consecuencias que tienen las intervenciones forestales en los bosques. Köhler (2002) informa que para los bosques de neblina en Costa Rica la biomasa de la vegetación epifita en un rodal viejo es mayor $\left(3,4 \mathrm{t} \mathrm{ha}^{-1}\right)$ que la de bosques secundarios $\left(0,2\right.$ a $\left.0,5 \mathrm{t} \mathrm{ha}^{-1}\right)$. Por lo tanto, los resultados del presente estudio confirman que en el caso de la Cordillera Pelada el criterio "bosque primario" o "bosque secundario" no es el adecuado para explicar la composición y abundancia de la vegetación epifita. Al contrario, entre el bosque primario de Laurelio-Weinmannietum y el bosque secundario de Drimys-Facies no se pudieron establecer diferencias en cuanto a la abundancia de las formas de vida de epifitas, ni en la biomasa. Sin embargo, el bosque secundario del Fitzroyetum se diferencia claramente de los otros bosques investigados. El factor clave para la diversidad y abundancia de las epifitas parece ser la estructura de los bosques y su habilidad de capturar neblina, y no sólo su condición de bosque primario o rodal secundario. La densidad del rodal, la composición arbórea y la presencia o ausencia de un sotobosque afectan las condiciones de luz y la humedad relativa del aire. Además, la composición arbórea determina con las características de la corteza de cada especie (ejemplo: la acidez o la habilidad de almacenar agua) la oferta de sitios para el crecimiento de las epifitas.

Una intervención dentro del manejo forestal, como cosecha o raleos, causa cambios en la estructura, densidad y composición arbórea de un rodal y con ello altera las condiciones para el desarrollo de las epifitas (Ingwerson 1985). El estudio muestra que no existen relaciones simples entre estas condiciones y el crecimiento de las epifitas. Por ejemplo, una mayor radiación solar puede inhibir el desarrollo de los helechos del género Hymenophyllum, pero fortalece el crecimiento de otros grupos, como es el caso de los líquenes fruticosos. Éstos prefieren lugares con una mayor exposición a la luz y, por consiguiente, muestran su mayor abundancia en rodales que registran mayor luminosidad.

El presente análisis comparativo de parcelas de bosques secundarios y de un bosque primario prueba que se pueden esperar cambios en la vegetación epifítica cuando se interviene en los bosques. No obstante, por la complejidad de las interrelaciones entre los distintos factores, no es posible predecir con exactitud su efecto sobre el crecimiento de las epifitas. Investigaciones sobre sus exigencias ecológicas y el crecimiento de ellas en los bosques son necesarias en el futuro para poder garantizar un manejo forestal sostenible de los bosques templados chilenos, que abarca tanto la conservación de la biodiversidad como el mantenimiento del balance hídrico y del ciclo de nutrientes.

\section{AGRADECIMIENTOS}

El estudio fue financiado por el Programa para el Fomento de Graduados de Baja Sajonia y el DAAD. Los autores expresan su agradecimiento a la CONAF, Provincia Valdivia, por las facilidades para trabajar en la Reserva Nacional Valdivia, en especial a Alex Rudloff, René Cárdenas y Laslo Pancel (GTZ). Además se agradece a los árbitros anónimos por sus comentarios.

\section{REFERENCIAS}

Alberdi M. 1966. Consideraciones generales sobre las turberas de la Cordillera Pelada. Boletín Universidad de Chile 65: 52-53.

Alberdi M, C Ramírez, L Steubing. 1978. La familia Hymenophyllaceae (Pteridophyta) en el fundo San Martín, Valdivia, Chile II. Resistencia al desecamiento y sobrevivencia en comunidades antropogénicas. Medio Ambiente 3(2): 3-13.

Albin R. 1975. Determinación del pH en diversas especies de los renovales de la provincia de Valdivia. Bosque 1(1): 3-5.

Aravena R, O Suzuki, A Pollastri. 1989. Coastal fog and its relation to groundwater in the IV region of northern Chile. Chem. Geol. 79: 83-91.

Armesto JJ, P León, M Kalin. 1997. Los bosques templados del sur de Chile y Argentina: una isla biogeográfica. En Armesto JJ, C Villagrán, M Arroyo eds. Ecología de los bosques nativos de Chile, Santiago de Chile. Editorial Universitaria. p. 23-28.

Bruijnzeel LA, EL Veneklass. 1998. Climatic conditions and tropical montane forests productivity: the fog has not lifted yet. Journal of Ecology 79: 3-9.

Cereceda P, R Schemenauer. 1991. The occurrence of fog in Chile. Journal of Applied Meteorology 30: 1097-1105.

Cisternas M, P Martínez, C Oyarzún, P Debels. 1999. Caracterización del proceso de reemplazo de vegetación nativa por plantaciones forestales en una cuenca lacustre de la Cordillera de Nahuelbuta, VIII Región, Chile. Rev. Chil. Hist. Nat. 72: 661-669.

Claro E. 1998. La influencia del mercado internacional de astillas en la destrucción de los bosques nativos chilenos. In Hoffmann A ed. La tragedia del bosque chileno. Santiago, Chile. Ocho Libros Editores. p. 311-316.

Dawson TE. 1998. Fog in the Californian redwood forest: ecosystem inputs and use by plants. Oecologia 117: 467-485.

Dohrenbusch A, J Kranigk, D Pryor. 1995. Entwicklung und Bau eines Lichtmeßgerätes zur Erfassung der photosynthetisch nutzbaren Strahlung. AFJZ 166(8): 109-114.

Echeverría C. 1994. Estudio comparativo de los componentes del balance hídrico de un bosque de Olivillo (Aextoxicon punctatum) y una pradera natural en el sur de Chile. Valdivia, Chile. Tesis Ingeniería Forestal, Facultad de Ciencias Forestales, Universidad Austral de Chile. 68 p.

Figueroa J, S Castro. 2000. Efecto de herbívoros y patógenos en la sobrevivencia y crecimiento de plántulas en un fragmento del bosque templado húmedo de Chiloé, Chile. Rev. Chil. Hist. Nat. 73: 163-173. 
Fuente J, J Hernández, J Caldentey, H Castillo. 1994. Efectos sobre la redistribución de las precipitaciones y los contenidos de humedad del suelo por la transformación de un bosque de Nothofagus obliqua a una plantación de Pinus radiata, en la Cordillera de Nahuelbuta. Ciencias Forestales 9(1-2): 23-39.

Galloway D. 1995. Los líquenes del bosque templado de Chile. In Armesto JJ, C Villagrán, M Arroyo ed. Ecología de los bosques nativos de Chile. Santiago, Chile. Editorial Universitaria. p. 101-112.

Godoy R, C Oyarzún, V Gerding. 2001. Precipitation chemistry in deciduous and evergreen Nothofagus forests of southern Chile under low-deposition climate. Basic and Applied Ecology 2: 65-72.

Grunow J. 1955. Der Niederschlag im Bergwald - Niederschlagszurückhaltung und Nebelzuschlag. Forstw. Cbl. 74: 21-36.

Guzmán G, W Quilhot, D Galloway. 1990. Decomposition of species Pseudocyphellaria and Sticta in a southern chilean forest. Lichenologist 22: 325-331.

Hall M, J Witte. 1998. Maderas del Sur de Chile. Santiago, Chile. IER Ediciones. 92 p.

Huber A. 1975. Beitrag zur Klimatologie und Klimaökologie von Chile. München, Alemania. Tesis Doctor. Forstliche Fakultät, Universidad de München. 87 p.

Huber A, C Oyarzún, M Oñate. 1986. Factores reguladores de la transpiración potencial de algunas especies arbóreas del bosque siempreverde del sur de Chile. Turrialba. 36(3): 329-336.

Huber A, A Martínez. 1995. Redistribución de las precipitaciones en un bosque de la pluviselva valdiviana. Medio Ambiente 12(2): 7-13.

Huber A, A Iroumé. 2001. Variability of annual rainfall partitioning for different sites and forest covers in Chile. Journal of Hydrology 248(1): 78-92.

Hueck K. 1966. Die Wälder Südamerikas. Vegetationsmonographien der einzelnen Großräume. In Walter $\mathrm{H}$ ed. Stuttgart, Alemania. Gustav Fischer Verlag. Vol. 2. 422 p.

Ingwerson JB. 1985. Fog drip, water yield and timber harvesting in the bull run municipal watershed, Oregon. Water Resources Bulletin 21(3): 469-473.

Köhler L. 2002. Die Bedeutung der Epiphyten im ökosystemaren Wasser- und Nährstoffumsatz verschiedener Altersstadien eines Bergregenwaldes in Costa Rica. Tesis Doctor. Göttingen, Alemania. Georg-August-Universität Göttingen.

Ramírez C, L Steubing, M Alberdi. 1976. La familia Hymenophyllaceae (Pteridophyta) en el fundo San Martín, Valdivia-Chile I. Taxonomía y ecología. Medio Ambiente 2(1): 21- 28.

Stadtmüller T. 1987. Cloud forests in the humid tropics. A bibliographic Review. Japan. The United Nations University. 82 p.
Steubing L, R Godoy. 1995. Verbreitung der Mykotrophie im Alerce-Wald und dessen Sekundärgesellschaften. Phytocoenologia 25(4): 579-593.

Subiabre A, C Rojas. 1994. Geografía física de la Región de Los Lagos. Valdivia, Chile. Universidad Austral de Chile. $117 \mathrm{p}$.

Oberdorfer E. 1960. Pflanzensoziologische Studien in Chile. In TÜXEN R, ed. Flora et Vegetatio Mundi, Weinheim, Alemania. Cramer. Vol. 2, p. 1-208.

Oyarzún C, R Godoy, A Sepúlveda. 1998. Water and nutrient fluxes in a cool temperate rainforest at the Cordillera de la Costa in southern Chile. Hydrological Processes 12: 1067-1077.

Oyarzún C, R Godoy, A De Schrijver, J Staelens, N Lust. 2004. Water chemistry and nutrient budget in an undisturbed evergreen rain forest of southern Chile. Biogeochemistry 71: 107-123.

Pérez C, J Armesto, B Ruthsatz. 1991. Decomposición de hojas, biomasa de raíces y características de los suelos en bosques mixtos de coníferas y especies laurifolias en el Parque Nacional Chiloé, Chile. Rev. Chil. Hist. Nat. 64: 479-490.

Steubing L, R Godoy, M Alberdi. 2002. Métodos de Ecología Vegetal. Santiago, Chile. Universitaria, 345 pp.

Veblen TT, D Ashton. 1982. The regeneration status of Fitzroya cupressoides in the Cordillera Pelada, Chile. Biological Conservation 23: 141-161.

Villagrán C, L Hinojosa. 1997. Historia de los bosques del sur de Sudamérica II: Análisis fitogeográfico. Rev. Chil. Hist. Nat. 70(2): 241-267.

Vogelmann HW. 1973. Fog precipitation in the cloud forests of eastern Mexico. Bioscience 23(2): 96-100.

Weathers KC, Lovett GM, Likens GE. 2000. Cloudwater inputs of nitrogen to forest ecosystems in southern Chile: forms, fluxes, and sources. Ecosystems 3(6): 590-595.

Weinberger P, M Romero, M Oliva. 1973. Untersuchungen über die Dürreresistenz patagonischer immergrüner Gehölze. Vegetatio 28(1-2): 75-98.

Weinberger P. 1974. Verbreitung und Wasserhaushalt araukano-patagonischer Protaceen in Beziehung zu mikroklimatischen Faktoren. Flora 163: 251-264.

Wilken P. 1998. La sustentabilidad forestal en Chile. Santiago de Chile: Serie documentos experiencias de cooperación $\mathrm{N}^{\circ}$ 3/98, DED - CODEFF. 62 p.

Wirth V. 1995. Die Flechten Baden-Würtembergs. Stuttgart. Eugen Ulmer. Vol. 2, 1006 p.

Woda C. 2004. Biomasse und Vegetationsstruktur von temperierten Regenwäldern in Chile (Cordillera Pelada). Forstarchiv 75(1): 3-12.

WWF. 2002. Ecorregiones globales. Consultado 1 dic. 2002. Disponible en: http: //wwww.panda.org/about_wwf/ where_we_work/ ecoregions/global200/pages/ endangered.htm. 